\title{
A Method for Detecting Undisplayed Regions in Virtual Colonoscopy and Its Application to Quantitative Evaluation of Fly-Through Methods
}

\author{
Yuichiro Hayashi ${ }^{1}$, Kensaku Mori ${ }^{1,2}$, Jun-ichi Hasegawa ${ }^{3}$, \\ Yasuhito Suenaga ${ }^{1}$, and Jun-ichiro Toriwaki ${ }^{1}$ \\ 1 Graduate School of Engineering, Nagoya University \\ Furo-cho, Chikusa-ku, Nagoya 464-8603, Aichi, Japan \\ \{yhayashi,mori, suenaga, toriwaki\}@toriwaki.nuie.nagoya-u.ac.jp \\ 2 Image Guidance Laboratories, Dept. of Neurosurgery, Stanford University \\ 300 Pasteur Drive Stanford, CA. 94305-5327, USA \\ ken@igl.stanford.edu \\ 3 School of Computer and Cognitive Sciences, Chukyo University \\ 101 Tokodachi, Kaizu-cho, Toyota 470-0393, Aichi, Japan \\ hasegawa@sccs. chukyo-u.ac.jp
}

\begin{abstract}
This paper shows a method for detecting regions that the system did not display during the fly-through of a virtual endoscope system (VES) and quantitative evaluation of undisplayed regions in virtual colonoscopy. When we use the VES as a diagnostic tool, especially as a tool for detecting colon polyps, a user often performs automated fly-through based on automatically generated paths. In the case of automated fly-through in the colon, there are some blind areas at the backs of folds. The aim of this study is to detect undisplayed regions during flythrough and to perform quantitative evaluation. After the fly-through, the system shows undisplayed regions and notifies the user of the existence of unobserved regions together with the rate of undisplayed regions. We evaluate various kinds of automated fly-through paths generated from medial axes of the colon and flattened views of the colon from the viewpoint of the rate of undisplayed regions. The experiment results show that about $30 \%$ of colon regions are classified as undisplayed regions by the conventional automated fly-through along the medial axis and that the flattened view results in very few undisplayed regions.
\end{abstract}

\section{Introduction}

Virtual endoscope systems (VESs) 1,23] are now widely used for observing the inside of a human body. A VES visualizes anatomical structures by generating virtual endoscopic (VE) images based on 3-D medical images such as CT or MR images. The user of a VES can observe the inside of an organ from any viewpoint and view direction. Virtual endoscopy can be used for various purposes such as diagnosis, pre-operative surgical planning, endoscopic navigation, and 
informed consent. Many research groups have reported applications of VE as screening tools of colorectal cancers (called virtual colonoscopy (VC)), since VC does not cause patients pain during examination procedures. colorectal cancer is the second leading cause of death in the U.S.A., and several companies are releasing software for $\mathrm{VC}$. In colorectal cancer screening using $\mathrm{VC}$, a medical doctor diagnoses the inside of the colon by observing VE views. When the inside of the colon is observed by using a VES for detection of polyps, fly-through techniques are usually used. Automated fly-through techniques are most frequently employed, since manual fly-through requires the doctor to change the viewpoint and the view direction of the virtual camera of the VES many times.

There are many studies on automated or semi-automated fly-through in VESs [2 34 4 5]. In these methods, fly-through paths are generated from the extraction results of the medial axes of colon regions. Then fly-through is performed along these paths. We also have developed a method for generating automated flythrough paths based upon medial axes generated by a thinning algorithm that uses the Euclidean distance transformation [5].

However, there are fold patterns called haustras in the colon. These fold patterns may cause some blind regions and oversighting of polyps. To reduce oversighting of colorectal polyps, the system should warn the user about regions that are not completely displayed on the screen (undisplayed regions). It is important to perform automated fly-through that does not result in undisplayed regions. Quantitative evaluation of undisplayed regions are also an important problem to solve. However, there is no report which describes quantitative evaluation of undisplayed regions of automated fly-through paths.

In this paper, we describe a method to detect undisplayed regions during flythrough of VC. It is based on novel simple techniques and is useful for improving diagnosis using VC. The concept of detection of unobserved and observed regions were first proposed by the authors' group in 2000 [5]. The detailed description of the method is presented in the reference [6]. Our concept and method has been referred to by another research group working on VC [7]. Our current paper now presents the quantitative evaluation of automated fly-through paths from the viewpoint of undisplayed regions by using the proposed method. In Section 2 , we describe the detection process of undisplayed regions during fly-through. Brief descriptions of several methods for creating automated fly-through paths in VC are given in the same section. In Section 3, we compare various automated fly-through methods according to the number of undisplayed regions detected. Discussion is also presented in the same section. Section 4 contains our conclusion.

\section{Methods}

\subsection{Detection of Undisplayed Regions}

Outline. This paper defines displayed regions as regions which are displayed at least once on a screen during the fly-through and undisplayed regions which are not displayed on the screen at all. Before the fly-through, the entire region of the colon is labeled as undisplayed. The basic idea of this method is to record 
displayed regions for every frame and to compute undisplayed regions from the record. During the fly-through, we record areas shown on the display for each frame of the VE views. This marking process is executed for all frames. At the end of the fly-through, regions not having displayed marks are counted as undisplayed regions. Surface rendering (SurR) and volume rendering (VolR) methods are often utilized for generating VE views. Since the minimum rendering units of SurR and VolR are a triangle and a voxel, respectively, displayed regions are marked by using these units.

Detection in SurR. In the SurR methods, the shape (surface) of the colon is represented as a set of triangles. These triangles are usually generated by the Marching Cubes method. VC images are obtained by rendering the triangles using suitable shading and projection techniques. Therefore we also classify the triangles into displayed and undisplayed ones during the fly-through. The method detects triangles displayed on the screen by the following steps. Prior to the fly-through, undisplayed marks are assigned to all triangles. For each frame of the VE views, we render two types of images: (a) shaded view and (b) labeled view. First, a normal VE view is generated with a proper shading procedure and displayed on the screen (shaded view). Then, we assign a different color to each triangle as the unique code of the triangle. Since these patches are rendered without shading, the color of each pixel of the screen shows the code of the displayed triangle (labeled view). This special rendering is performed on the back-buffer (frame buffer that is not currently displayed on the screen), with which conventional graphics hardware is equipped. We mark all of the triangles detected in this process as displayed. At the end of the fly-through, triangles not having displayed marks are classified into undisplayed triangles. A set of connected undisplayed-triangles are considered as undisplayed regions.

Detection in VolR. In the VolR method, we extract undisplayed regions as a set of voxels which are not projected on the screen. Basically, VolR is performed by casting a ray that starts at the viewpoint and passes through the pixel on the projection screen. Prior to the rendering process, we assign the color and opacity values to each voxel of an input 3-D image. Weighted accumulation of the shaded color of each voxel is performed along the cast ray. Each pixel value of the rendered image is computed by weighted-accumulation operations. The voxel having the highest accumulated-opacity value dominates the color of the screen pixel for each ray. Therefore, we consider voxels existing between the voxel with the maximum accumulated opacity value and the viewpoint as displayed voxels. The detection process is implemented in the following manner. First, all voxels of an input image are marked as undisplayed. During the rendering process for generating virtual endoscopic views, we also simultaneously perform a checking process of displayed voxels by using the above method. This process is executed for all frames of the fly-through. 


\section{$2.2 \quad$ Automated Fly-Through}

Automated Fly-through Along the Medial Axis (Method A). This method continuously changes the viewpoint and the view direction of the virtual camera of the VES along the medial axes of colon regions [5]. Many others research groups have reported on automated generation of fly-through paths based on medial axes of the colon [3/4]. Basically, these methods obtain medial axes of colon regions by applying thinning methods. In this paper, we extract medial axes of the colon by applying a thinning method that uses the result of the Euclidean distance transformation as in 5]. The fly-through path is computed by specifying two points (start and end points) on the thinned result. Viewpoints are allocated on the obtained path at a predefined interval. The tangent direction of the path at each viewpoint is used for the view direction at the point.

\section{Modified Fly-through by Using Haustra Information (Method B).} Since there are many folds in the colon, it is impossible to observe the colonicwall regions existing between the folds. This method modifies the path obtained in Method A so that the virtual camera rotates 360 degrees to visualize the colonic walls between fold patterns when it comes among the folds. Fold regions are extracted by applying morphological and connected component operations to the extracted colon region.

Flattened View. For comparison, we also generate flattened views of the colon along the path obtained in Method A. The flattened view is generated by modifying the paths of ray casting of the VolR. At each viewpoint along the fly-through path, we cast rays to cover 360 degrees in a direction perpendicular to the path and get one line of the rendered image (called a rendered line). We execute this casting process on each point of the path and stack the rendered lines to obtain the final image.

\section{Experiments and Results}

\subsection{Materials}

We implemented the above-mentioned methods on a conventional PC (CPU: Intel Pentium $41.70 \mathrm{GHz}$, OS: Windows 2000, Memory: 1 Gbyte) and applied them to three colon $\mathrm{CT}$ data sets. Colon regions were extracted from a 3-D abdominal X-ray CT image by using a region growing method with a position variant thresholding operation. Acquisition parameters of the CT image were: $0.625 \sim 0.684 \mathrm{~mm}$ pixel size, $512 \times 512$ pixels, $112 \sim 349$ slices, $5.0 \sim 2.5 \mathrm{~mm}$ slice thickness, and $2.0 \sim 1.25 \mathrm{~mm}$ reconstruction pitch. We generated many paths for automated fly-through and calculated the rates of undisplayed regions over the whole regions for the paths. A flattened view of the colon was also generated for comparison. 


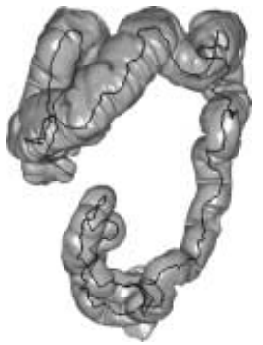

(a)

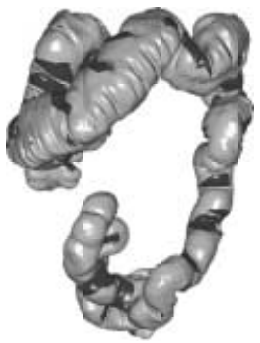

(b)

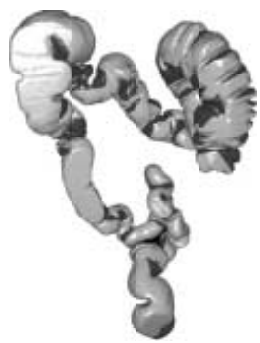

(c)

Fig. 1. Automated fly-through path along the medial axis of colon and detected undisplayed regions. (a) Fly-through path overlaid on the outside view of colon with black solid line, (b) and (c) Uudsplayed regions. Detected regions are marked with dark color.

Table 1. Experiment results of computation of RUD (the rate of undisplayed regions to the whole regions in \%).

\begin{tabular}{|c|c|c|c|c|c|c|c|}
\hline & $\begin{array}{c}\text { Movement } \\
\text { (voxels) }\end{array}$ & \multicolumn{2}{c|}{$x$-direction } & \multicolumn{2}{c|}{-direction } & \multicolumn{2}{c|}{$z$-direction } \\
\cline { 2 - 8 } & SurR & VolR & SurR & VolR & SurR & VolR \\
\hline \hline \multirow{3}{*}{ Case 1 } & 0 & 37.6 & 29.6 & 37.6 & 29.6 & 37.6 & 29.6 \\
\cline { 2 - 8 } & +5 & 37.5 & 30.0 & 37.9 & 30.2 & 36.1 & 28.3 \\
\cline { 2 - 8 } & -5 & 37.6 & 29.8 & 37.5 & 29.3 & 39.1 & 31.3 \\
\hline \multirow{4}{*}{ Case 2 } & 0 & 27.4 & 21.0 & 27.4 & 21.0 & 27.4 & 21.0 \\
\cline { 2 - 8 } & +5 & 27.2 & 21.0 & 27.0 & 20.5 & 26.5 & 20.1 \\
\cline { 2 - 8 } & -5 & 27.4 & 20.9 & 27.6 & 21.2 & 28.0 & 21.7 \\
\hline \multirow{3}{*}{ Case 3 } & 0 & 29.4 & 21.1 & 29.4 & 21.1 & 29.4 & 21.1 \\
\cline { 2 - 8 } & +5 & 27.5 & 20.8 & 29.0 & 21.0 & 28.4 & 20.2 \\
\cline { 2 - 8 } & -5 & 29.4 & 21.5 & 29.6 & 21.4 & 29.8 & 22.0 \\
\hline
\end{tabular}

We define the rate of undisplayed regions to displayed regions (RUD) in the following way. For SurR, RUD is computed as (Number of undisplayd patches) / (Total number of triangle patches). The RUD for VolR is defined as (Number of undisplayed voxels on colonic wall) / (Total number of voxels on colonic wall). we define voxels on the colonic wall in the following way. First, we apply the fusion operation (shrink and expand type) to the colonic regions extracted by using the region growing method. Voxels on the colonic wall are determined as voxels which are connected to background voxels in six-neighborhood connectivity.

\subsection{Results and Discussion}

We evaluated the ratios of undisplayed regions for automated fly-through paths based on the medial axes of the colon (Method A). we also translated the paths in parallel to three axes of the CT image coordinate systems within \pm 5 voxels and computed the RUDs. This is because we consider that the paths generated by other research groups' methods are located within \pm 5 voxels of our paths. Figure 1 shows examples of the fly-through path and undisplayed regions detected by the 
Table 2. RUD (in \%) with changed the intervals between the viewpoints in automated fly-through.

\begin{tabular}{|c|c|c|c|c|c|c|}
\hline \multirow{2}{*}{$\begin{array}{c}\text { Interval } \\
\text { (voxels) }\end{array}$} & \multicolumn{2}{|c|}{ Case 1 } & \multicolumn{2}{c|}{ Case 2 } & \multicolumn{2}{c|}{ Case 3 } \\
\hline \hline 1 & 37.6 & 29.6 & 27.4 & 21.0 & 29.4 & 21.1 \\
\hline 0.5 & 37.2 & 29.5 & 27.1 & 20.8 & 29.3 & 20.9 \\
\hline 0.1 & 36.8 & 29.3 & 26.7 & 20.7 & 28.6 & 20.7 \\
\hline
\end{tabular}

Table 3. RUD (in \%) with changed FOV angles.

\begin{tabular}{|c|c|c|c|c|c|c|}
\hline \multirow{2}{*}{$\begin{array}{c}\text { FOV } \\
\text { (degrees) }\end{array}$} & \multicolumn{2}{|c|}{ Case 1 } & \multicolumn{2}{c|}{ Case 2 } & \multicolumn{2}{c|}{ Case 3 } \\
\hline \hline 70 & 42.2 & 34.5 & 37.1 & 25.0 & 33.7 & 25.2 \\
\hline 80 & 37.2 & 29.6 & 27.4 & 21.0 & 29.4 & 21.1 \\
\hline 90 & 33.4 & 25.4 & 23.3 & 17.1 & 25.4 & 17.6 \\
\hline 100 & 29.6 & 21.7 & 19.6 & 13.8 & 21.8 & 14.5 \\
\hline 110 & 26.1 & 18.6 & 16.1 & 10.7 & 18.4 & 11.6 \\
\hline
\end{tabular}

proposed method in the case of zero-translation of the path. In Fig.1 (b) and (c), undisplayed regions are marked with dark color. There exist many undisplayed regions between the folds. Table 1 shows RUD for paths translated along each axis. For all paths, about $30 \%$ of regions were detected as undisplayed. In the experiments on parallel translation of the paths, RUDs were almost the same rate. There is no significant difference between SurR and VolR methods. This means that there is the possibility that the automated fly-through methods proposed by many research groups including the authors' group [5] may result in many undisplayed regions.

We also changed the interval of viewpoints in the generation process of the automated fly-through path and computed RUDs for each interval. In the experiment, we set the interval of the viewpoint as $1,1 / 2$, and 1/10 of the voxel size. The results are shown in Table 2 . If we shorten the interval of the viewpoint, RUD is slightly decreased. However, the decrease in RUD is within 1\%. This means that shortening of the viewpoint interval is not an appropriate solution for reducing the rate of undisplayed regions.

In VC, the appropriate choice of FOV (field of view) angle is one of the most important problems to solve. We investigated the relation between RUD and FOV. The FOV angle was changed from 70 degrees to 110 degrees with 10 degree intervals. The RUD was calculated for each FOV angle by using the automated fly-through path of Method A. The experimental results are shown in Table 3. As expected, wider angle FOV clearly reduces RUD. Although wider angle FOV reduces RUD, it causes VE views with high perspective-distortion. Highly distorted images makes the diagnosis process difficult. This is a trade-off for reduction of RUD.

Round-trip paths are effective methods for reducing RUD in VC. In a roundtrip path, the virtual camera of the VES moves from the start point to the end 
Table 4. RUD (in \%) for one way path and round-trip path.

\begin{tabular}{|c|c|c|c|c|c|c|}
\hline & Case 1 & \multicolumn{2}{c|}{ Case 2 } & \multicolumn{2}{c|}{ Case 3 } \\
\cline { 2 - 7 } & SurR & VolR & SurR & VolR & SurR & VolR \\
\hline \hline One-way path & 37.6 & 29.6 & 27.4 & 21.0 & 29.4 & 21.1 \\
\hline Round-trip path & 17.1 & 12.1 & 7.6 & 4.7 & 9.0 & 6.0 \\
\hline
\end{tabular}

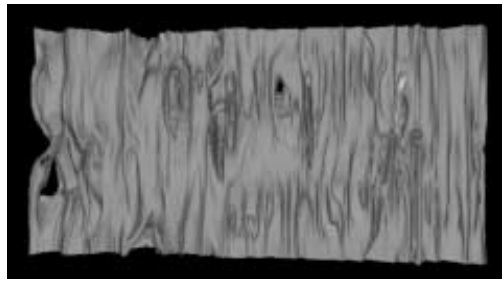

(a)

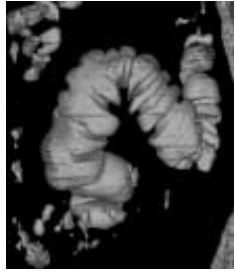

(b)

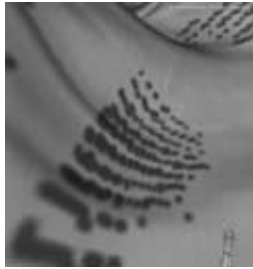

(c)

Fig. 2. Example of flattened views and detection results of undisplayed regions. (a) Flattened view, (b) and (c) undisplayed regions. Detected regions are marked with dark color.

point along the automated fly-through path. Then at the end point, the virtual camera changes its viewing direction by 180 degrees and follows the path back toward the start point. We quantitatively evaluated the difference between the one-way path and the round-trip path. Table 4 shows the experiment results. These results show that the round-trip path is a very effective way to reduce the rate of undisplayed regions if the double of examination is acceptable.

Method B described in Section 2.2 forces the virtual camera to face the colonic wall during fly-through to prevent regions between folds from becoming undisplayed. In the experiment result for Case 1, the RUDs of Method B were $13.5 \%$ for SurR and $9.0 \%$ for VolR, while the conventional path (Method A) resulted in about 30\% RUD for both SurR and VolR. Therefore, the use of fold pattern information in the process of creating fly-through paths is quite effective.

Several research groups have proposed the methods for generating flattened views of the colon. Our interest here is the extent of the reduction of RUD that can be achieved by introducing the flattened view. The proposed method can quantitatively evaluate and compare undisplayed regions for both visualization techniques. Examples of the flattened view and detection results are shown in Fig. 2. In the flattened views of Case 1, the RUD value is $2.7 \%$ for VolR. This shows flattened views of the colon are quite effective for observation of the status of the colonic wall while keeping RUD low. Although the user can easily review the colon by only one or a few stretched views, the rendered views contain heavy distortion. This cause the medical doctor some difficulty when diagnoseing from these flattened views. 


\section{Conclusion}

This paper proposed methods for detecting undisplayed regions in VC. We also compared automated fly-through methods from the viewpoint of the ratio of undisplayed regions. Hither to, there has been no published article about detection and quantitative evaluation of undisplayed regions for VC. Basically, undisplayed regions are detected by marking displayed triangles for SurR or displayed voxels for VolR. We consider the voxels or triangles not having displayed marks as undisplayed triangles or voxels. We also quantitatively evaluated several automated fly-through techniques by using the proposed method. The flattened view was also employed for comparison. The experiment results showed that the RUD of the conventional automated fly-through is about 30\%. The RUD of the automated fly-through is much decreased by using haustra information. The flattened view shows quite low RUD values and this visualization technique is therfor useful for diagnosis of the colon.

\section{Acknowledgments}

The authors would like to thank our colleagues for useful suggestions and discussion. K. Mori thanks Dr. Ramin Shahidi and Dr. Calvin Maurer, Jr., for providing him with opportunity to write this paper. Parts of this research were supported by the Grant-In-Aid for Scientific Research from the Japan Society for Promotion of Science, and the Grant-In-Aid for Cancer Research from the Ministry of Health, Labour and Welfare of the Japanese Government.

\section{References}

1. P. Rogalla, J. Terwisscha van Scheltinga, B. Hamm, eds., "Virtual endoscopy and related 3D techniques," Springer, Berlin, 2001.

2. K. Mori, A. Urano, J. Hasegawa, et al., "Virtualized endoscope system -an application of virtual reality technology to diagnostic aid-," IEICE Trans. Inf. \& Syst., vol. E79-D, no. 6, pp. 809-819, 1996.

3. L. Hong, S. Muraki, A. Kaufman, et al., "Virtual voyage: interactive navigation in the human colon," Computer Graphics (Proc. of Siggraph'97), pp. 27-34, 1997.

4. D.S. Paik, C.F. Beaulieu, R.B. Jeffrey, et al., "Automated path planning for virtual endoscopy," Medical Physics, vol. 25, no. 5, pp. 629-637, 1998.

5. Y. Hayashi, K. Mori, T. Saito, et al., "Advanced navigation diagnosis with automated fly-through path generation and presentation of unobserved regions in the Virtualized Endoscope System," Proc. of MIRU2000, pp. 331-336, 2000 (in Japanese).

6. K. Mori, Y. Hayashi, Y. Suenaga, et al., "A method for detecting unobserved regions in virtual endoscopy system," Proc. of SPIE, Medical Imaging, vol. 4321, pp. 134-145, 2001.

7. K. Kreeger, F. Dachille, M.R. Wax, et al., "Covering all clinically significant areas of the colon surface in virtual colonoscopy," Proc. of SPIE, Medical Imaging, vol. 4683, pp. 198-206, 2002. 